\section{Review of fundus autofluorescence in choroidal melanocytic lesions}

K Gündüz ${ }^{1,3}$, JS Pulido', K Ezzat'1, D Salomao ${ }^{2}$ and C Hann ${ }^{2}$

\section{Abstract}

Fundus autofluorescence (FAF) imaging takes advantage of the fluorescent properties of some molecules, especially lipofuscin. FAF derives mainly from retinal pigment epithelium (RPE) and Bruch's membrane. Using confocal scanning laser ophthalmoscope (cSLO) we have previously shown that FAF associated with pigmented choroidal lesions can be attributed to mainly lipofuscin (orange pigment) within the RPE. Other causes of FAF include hyperpigmentation, drusen, or fibrous metaplasia probably because they also cause lipofuscin accumulation in the overlying RPE. There is a total or partial correlation between FAF and the foci of lipofuscin and hyperpigmentation in about $90 \%$ of the cases. The FAF patterns of choroidal melanocytic lesions were classified as patchy or diffuse. The patchy pattern was defined as the presence of distinct areas of increased FAF between areas of normal autofluorescence. The diffuse pattern was characterized by the presence of increased FAF with indistinct borders over a larger part ( $>50 \%$ ) of the tumour in the absence of such intervening areas. Choroidal melanomas presented with either a diffuse or patchy pattern, whereas choroidal naevi demonstrated only the patchy pattern. Diffuse FAF pattern was more often associated with larger choroidal melanomas as well as with early venous and late hyperfluorescence on fluorescein angiography. Limitations of these observations depend on the field of depth of cSLO; thus, FAF from other planes could not be detected. Increased retinal thickness, intraretinal oedema, or presence of subretinal fluid may also affect the FAF signal. Eye (2009) 23, 497-503; doi:10.1038/eye.2008.244; published online 1 August 2008
Keywords: fundus autofluorescence; choroidal melanoma; choroidal naevus; angiography; lipofuscin; optical coherence tomography

\section{Introduction}

Fundus autofluorescence (FAF) photography relies on the stimulated emission of light from naturally occurring fluorophores, the most significant being lipofuscin. ${ }^{1-3}$ Lipofuscin is composed of lipids, proteins, and $\mathrm{N}$-retinyl- $\mathrm{N}$ retinylidene ethanolamine; the last component is considered a by-product of phagocytosis of membranous discs shed from the outer segments of photoreceptors. It is believed that the accumulation of lipofuscin is secondary to an impaired or exaggerated lysosomal activity with incomplete degradation. ${ }^{4} \mathrm{~A}$ fraction of lipofuscin can also exist bound to melanin.

Different patterns of FAF have been described in many retinal and choroidal diseases, and have been extensively studied in age-related macular degeneration (ARMD). Previous studies have extensively described changes of FAF in eyes with dry ARMD. Increased FAF was found to correlate with areas of

hyperpigmentation, soft drusen, hard drusen, or normal fundus appearance. ${ }^{5-8}$ Drusen may have an increased, normal, or decreased FAF signal. ${ }^{6}$ Overall, larger soft drusen were more often associated with increased FAF than smaller ones. ${ }^{6}$ Several studies concluded that FAF imaging in dry ARMD does not necessarily correspond to the fundus changes observed and provides information over and above the normal fundus photography methods used. ${ }^{6,9}$

Recently, FAF changes in wet AMD were also studied. One study showed that classic choroidal neovascular membrane (CNVM) is associated with decreased FAF, presumably because of its localization above the retinal
${ }^{1}$ Department of Ophthalmology, Mayo Clinic College of Medicine, Rochester, MN, USA

${ }^{2}$ Department of Pathology, Mayo Clinic College of Medicine, Rochester, MN, USA

${ }^{3}$ Present address: K Gündüz is now Professor of Ophthalmology at Department of Ophthalmology, Faculty of Medicine, Ankara University. Correspondence: K Gündüz, Department of Ophthalmology, Faculty of Medicine, Ankara University, Dikimevi 06590, Ankara,

Turkey

Tel: + 905326337997 ;

Fax: + 903122291812

E-mail: eyemd@

ada.net.tr

Received: 27 December 2007

Accepted in revised form: 13 May 2008

Published online: 1 August 2008

None of the authors have any proprietary interest in any of the products mentioned in this paper. 
pigment epithelium (RPE) level leading to blockage of FAF. ${ }^{10}$ Occult CNVM has a regular or mottled FAF pattern. ${ }^{10}$ Another study found that FAF imaging characteristics depend on the duration of CNVM. ${ }^{11}$ Recent-onset lesions ( $<1$ month) are associated with increased FAF, whereas chronic lesions $(>1$ month after onset) have decreased FAF. ${ }^{11}$

FAF studies in acute central serous chorioretinopathy reveal increased FAF in the active stages corresponding to increased metabolic activity of the RPE, and decreased FAF in chronic stages indicating reduced metabolic activity of the RPE because of photoreceptor cell loss. ${ }^{12}$ Hereditary retinal dystrophies usually present with increased FAF, although exceptions exist. ${ }^{13,14}$ RPE atrophy and cell loss, as in geographic atrophy or pseudoxanthoma elasticum, have been associated with decreased FAF. ${ }^{15,16}$ FAF has also been used to study the success of selective RPE destruction by laser treatment. Initial hypoautofluorescence observed after RPE destruction by laser treatment was found to change to hyperautofluorescence after 1 week because of the proliferation of RPE. ${ }^{17}$

Lipofuscin pigment accumulates in RPE cells and macrophages over several types of benign and malignant choroidal tumours. ${ }^{18,19}$ Data from fluorescence microscopic examination of choroidal melanomas show that the tumour itself does not show significant fluorescence. FAF derives from RPE and Bruch membrane, as the signal appears to originate from anterior to the choriocapillaris and posterior to the neuroretina. Increased fluorescence in enlarged lipofuscin-filled RPE adjacent to the normal retina account for the fluorescence observed in choroidal melanomas (Figures 1 and 2).

In a previous study, we looked at the correlation of increased FAF patterns with changes present on the surface of such lesions, including lipofuscin (orange pigment), hyperpigmentation, drusen, and fibrous metaplasia, in 23 cases with choroidal melanocytic lesions. ${ }^{20}$ Autofluorescent fundus photography was performed using the Heidelberg confocal scanning laser ophthalmoscope (cSLO) system (Heidelberg Retina Angiograph (HRA); Heidelberg Engineering, Dossenheim, Germany). In this machine, autofluorescence (AF) is excited by the argon blue wavelength $(488 \mathrm{~nm})$, and the emitted light above $500 \mathrm{~nm}$ is detected with a barrier filter. Maximal retinal irradiation at $30^{\circ}$ is $0.2 \mathrm{~mW} / \mathrm{cm}^{2}$, well below the limits established by the American National Standards Institute. ${ }^{21}$ The confocal detection unit uses a $400 \mu \mathrm{m}$ pinhole aperture to eliminate the light from below or above the confocal plane. Patients with media opacities, including cataract, opacified posterior capsule, asteroid hyalosis, vitreous debris, and vitreous haemorrhage,

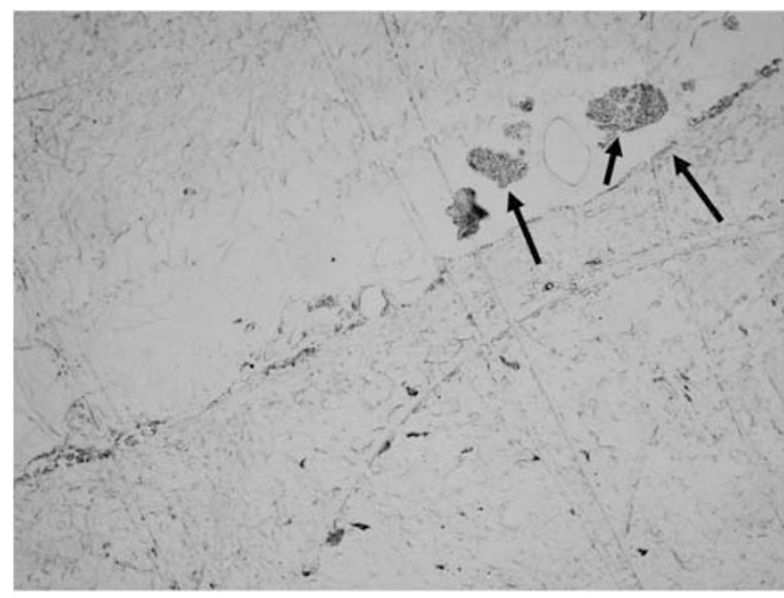

Figure 1 Unstained fixed section of a choroidal melanoma. There are large irregular RPE cells (arrows) filled with lipofuscin separating the retina (upper portion of the photograph) and the underlying melanoma $(\times 20)$.

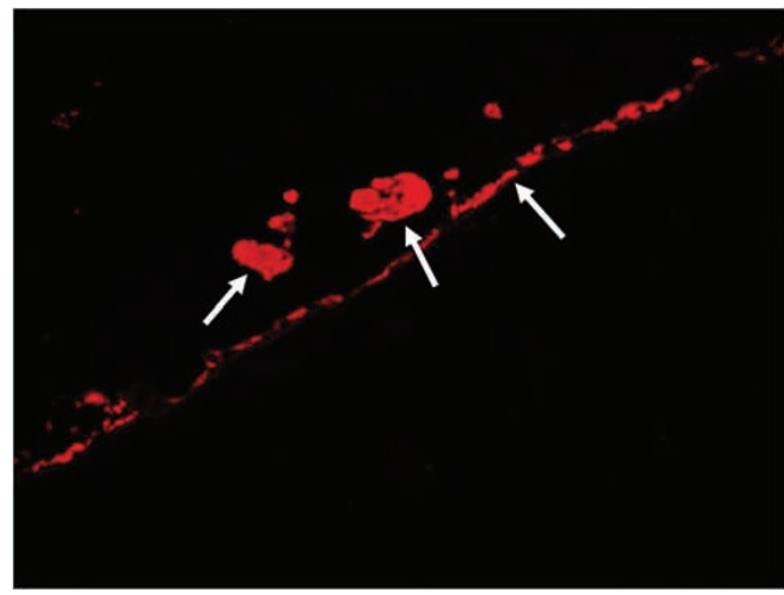

Figure 2 Corresponding autofluorescence photomicrograph showing marked autofluorescence of the swollen RPE cells and autofluorescence from the surrounding normal RPE (arrows). No autofluorescence is noted from the underlying melanoma $(\times 20)$.

were excluded from the study, as these factors may interfere with the quality of the FAF images. Patients who had received earlier ocular treatment were also excluded.

Color fundus photographs were carefully evaluated for the presence and location of lipofuscin,

hyperpigmentation, drusen, and fibrous metaplasia.

Similarly, FAF images were evaluated for the presence of areas of increased AF corresponding to the location of lipofuscin, hyperpigmentation, drusen, or fibrous metaplasia. The correlation between clinical photographs and FAF images was evaluated as complete correlation, 

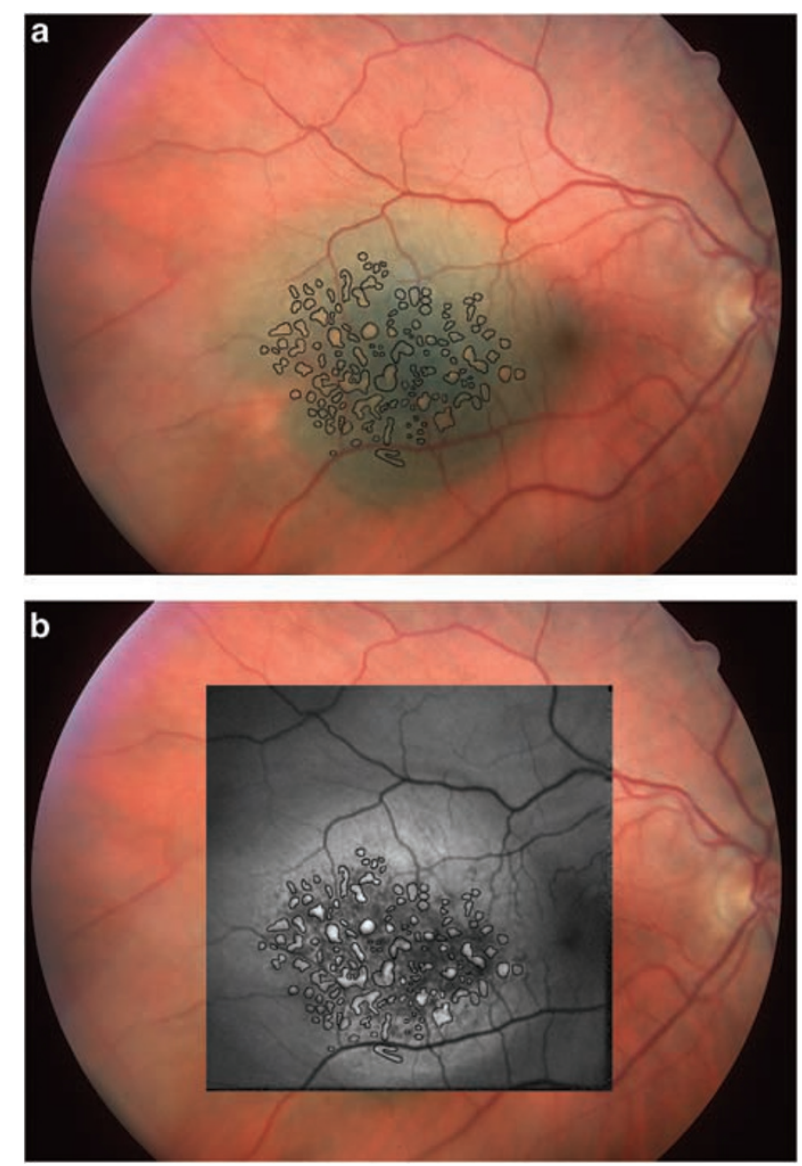

Figure 3 (a) Colour fundus photograph of a pigmented choroidal melanoma demonstrating several foci of lipofuscin overlying the lesion (black outlines). (b) The composite FAFcolour fundus picture of the same eye demonstrating increased FAF patterns corresponding to the location of lipofuscin (black outlines).

partial correlation, or no correlation separately for each of the four variables, whichever were present in a particular lesion. ${ }^{20}$

A complete correlation was defined as the presence of increased FAF corresponding to all areas of a clinically observed variable. A partial correlation was defined as the presence of at least one area of focally increased FAF corresponding to at least one area of a clinically observed variable in the absence of complete correlation. No correlation was defined as a complete lack of correspondence between any of the clinically observed features and FAF images for a given variable.

On the basis of the criteria outlined above, the location of lipofuscin (orange pigment) and/or

hyperpigmentation corresponded exactly to the location of increased FAF in $50 \%$ of the choroidal melanocytic tumours (Figures $3 a, b$ and $4 a, b) .{ }^{20}$ In another $40 \%$ of the cases, there was partial correlation between increased FAF and the location of orange pigment and/or
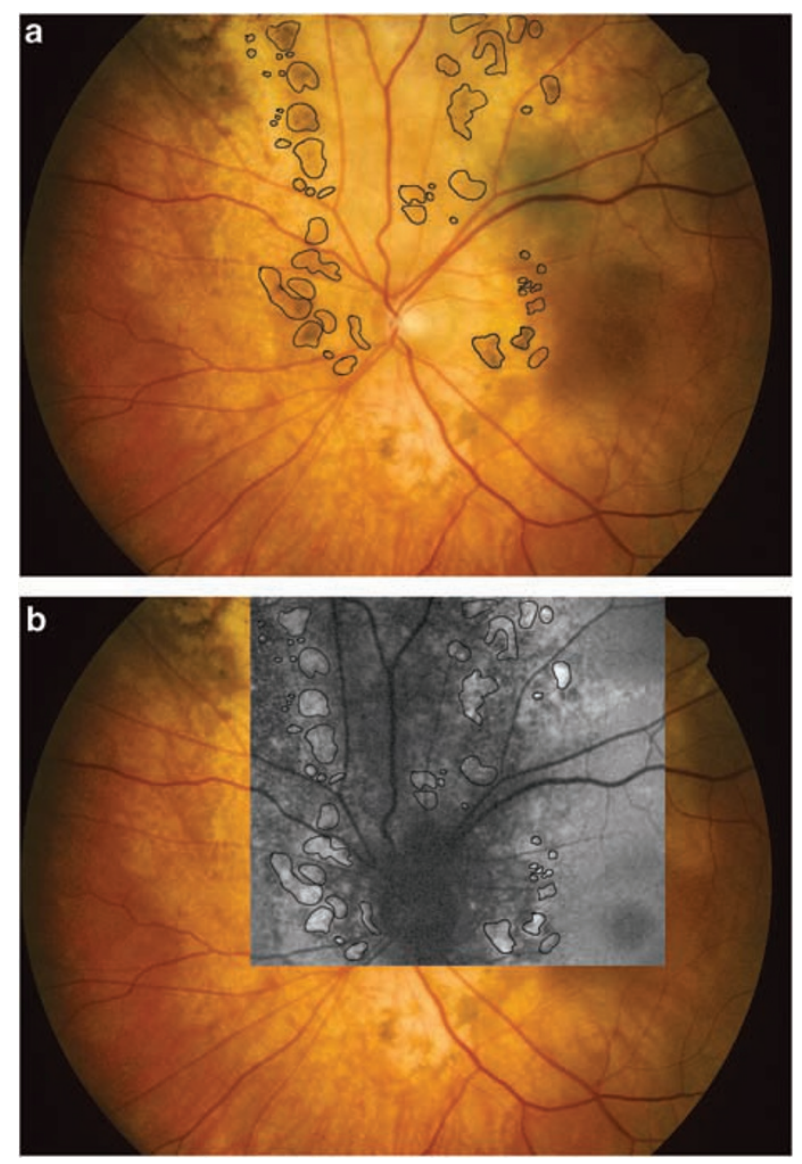

Figure 4 (a) Colour fundus photograph of an amelanotic choroidal melanoma showing several foci of hyperpigmentation on the lesion surface (black outlines). (b) The composite FAFcolour fundus picture of the same eye demonstrating increased FAF patterns at the sites of hyperpigmentation (black outlines).

hyperpigmentation..$^{20}$ Therefore, in $90 \%$ of the cases, there was either a total or partial correlation between increased FAF and the location of orange pigment and/ or hyperpigmentation. In amelanotic choroidal melanocytic lesions, the orange pigment had a more brown color because of the light background. We observed increased FAF intensities in these areas as well.

In this study of 23 eyes with choroidal melanocytic lesions, the presence of drusen and fibrous metaplasia showed partial correlation with increased FAF. ${ }^{20}$ Another study found that FAF in choroidal melanomas also correlated with fibrous metaplasia. ${ }^{22}$ It is known that collagen may be associated with $\mathrm{AF}$, although the excitation and emission spectra are usually different from the ones used in studying $\mathrm{FAF}^{23}$ It is possible that the increased FAF in fibrous metaplasia may be secondary to its collagen content in addition to RPE-associated lipofuscin. The role of FAF in other forms of choroidal tumours, such as choroidal metastasis, is presently under 
investigation (Collet L, Pulido JS, Gündüz K, unpublished observations)

The use of FAF imaging may have an important impact on clinical decision-making. The decision to treat choroidal melanocytic lesions depends on the presence of risks for growth, including lipofuscin, subretinal fluid, tumour thickness, proximity to optic nerve, and visual symptoms. ${ }^{24,25}$ Most ocular oncologists will treat a choroidal lesion if two or more of these five risk factors for growth are present, as the risk for growth in 5 years is above $50 \% .^{24}$ Furthermore, documented growth is a risk factor for metastasis. ${ }^{25}$ Under these circumstances, the assessment of lipofuscin becomes a crucial issue. Clinically, the lipofuscin pigment is seen as orangecoloured patches over a pigmented choroidal melanocytic lesion. The presence of subtle lipofuscin pigment in choroidal melanocytic lesions can be more difficult to assess. FAF imaging can be particularly helpful in these situations to demonstrate the lipofuscin content of the lesion. Furthermore, the determination of lipofuscin over amelanotic lesions can be even more challenging. Our study showed that hyperpigmentation over amelanotic choroidal lesions may actually represent accumulations of lipofuscin or melanolipofuscin and, therefore, should be considered as an important factor in the decision-making process for treatment.

In another study, we analysed the correlations between the FAF features and the fluorescein/indocyanine green angiographic (FA/ICGA) findings in 30 choroidal melanocytic lesions, including 19 choroidal melanomas and 11 choroidal naevi. ${ }^{26}$ Fundus fluorescein and indocyanine green angiographies were performed using the Heidelberg cSLO system. The colour fundus, AF, fluorescein, and indocyanine green angiography images were evaluated by two different observers to confirm interobserver variability. Partially inspired by previous studies, the FAF patterns in choroidal melanocytic lesions were tentatively classified as patchy and diffuse. ${ }^{27}$ The patchy pattern was defined as the presence of distinct areas of increased FAF between areas of normal AF. The diffuse pattern was characterized by the presence of increased FAF with indistinct borders over a larger part $(>50 \%)$ of the tumour in the absence of such intervening areas. The 19 choroidal melanomas included

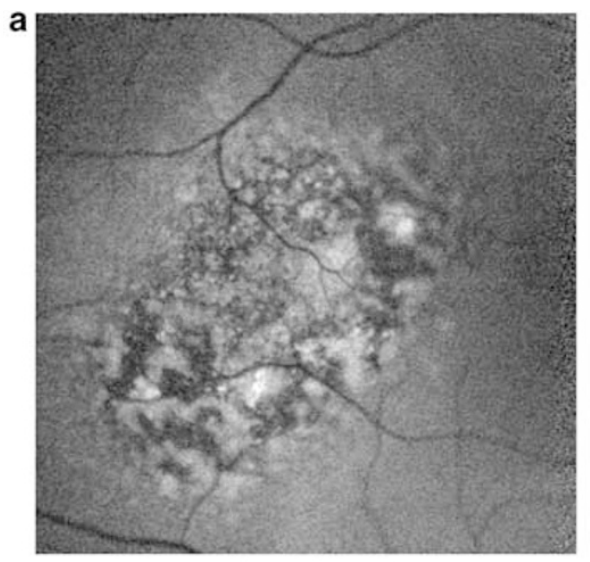

b

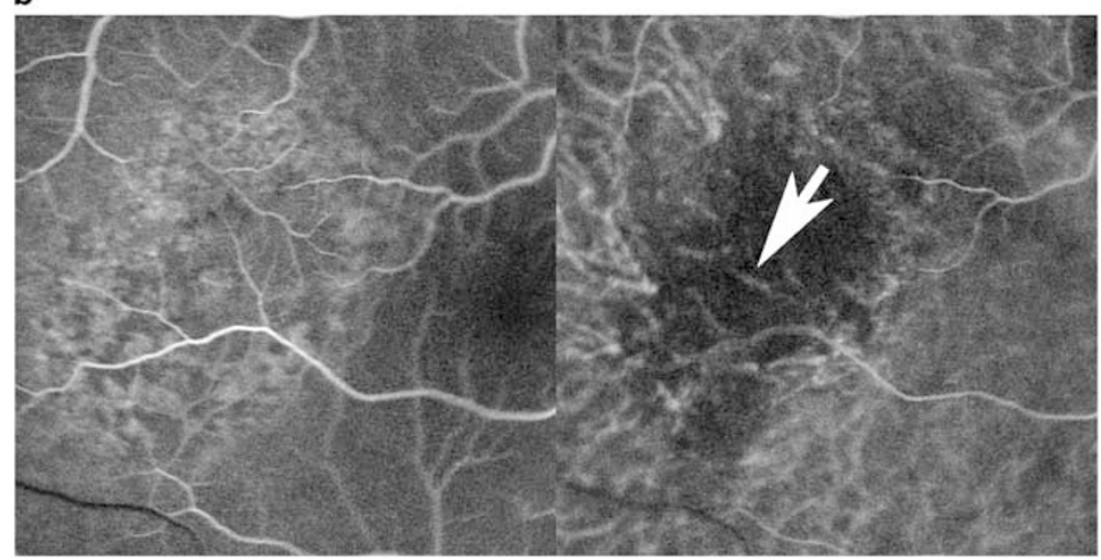

Figure 5 (a) Fundus autofluorescence image of the choroidal melanoma showing a diffuse pattern. (b) Early venous phase FA showing hyperfluorescence in the choroidal melanoma (left). Intrinsic tumour vessels are seen on ICGA (arrow) (right). 
in this study had either a diffuse or patchy FAF pattern, whereas the 11 choroidal naevi had the patchy FAF pattern. Tumours demonstrating a diffuse pattern can be expected to harbour a diffuse spread of lipofuscin/ melanolipofuscin over the lesion surface. Consistent with this expectancy, all tumours with a diffuse FAF pattern were choroidal melanomas. The mean tumour thickness was $5.3 \mathrm{~mm}$ and the mean largest tumour base was $12.6 \mathrm{~mm}$ in this group comprising 13 choroidal melanomas. The six choroidal melanomas with a patchy FAF pattern had a mean tumour thickness of $2.5 \mathrm{~mm}$ and a mean largest tumour base of $9.3 \mathrm{~mm}$. Therefore, larger and thicker choroidal melanomas may preferentially demonstrate a diffuse FAF pattern. ${ }^{26}$

In FA, most choroidal melanomas with a diffuse FAF pattern were hyperfluorescent starting in the early venous phase of the FA (Figure $5 a$ and b). ${ }^{26}$ These tumours remained hyperfluorescent throughout the late frames as well. Choroidal melanomas with a patchy FAF pattern usually demonstrated later hyperfluorescence compared with those with a diffuse pattern (Figure 6a and b). The hyperfluorescence in choroidal melanomas with a patchy pattern was usually evident in the mid-late phase (Figure 6a and b). Choroidal naevi demonstrated fluorescein angiographic findings similar to choroidal melanomas with a patchy pattern. ${ }^{26}$

In ICGA, most choroidal melanomas remained hypofluorescent/isofluorescent during the entire angiogram. Most choroidal naevi were hypofluorescent. Intrinsic tumour vessels were visible in 27 of 30 tumours with the exception of 3 choroidal naevi. Similar findings have been reported before concerning ICGA findings of choroidal melanomas and choroidal naevi. ${ }^{28}$

Using Fisher's exact test, the diffuse FAF pattern was significantly associated with the clinical diagnosis of choroidal melanoma $v$ s choroidal naevus $(P=0.00001)$, partially pigmented or amelanotic $v$ s pigmented lesion colour $(P=0.006)$, early venous hyperfluorescence on FA $(P=0.015)$, and late hyperfluorescence on FA $(P=0.018) .{ }^{26}$ Using two sample $t$-tests, the diffuse FAF pattern was significantly associated with increased

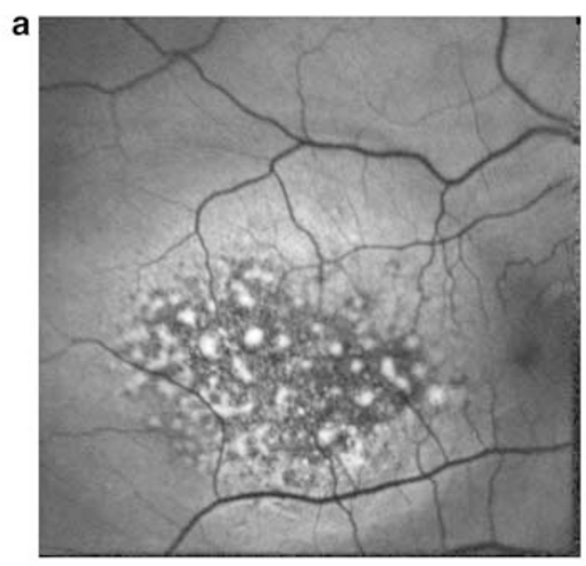

b

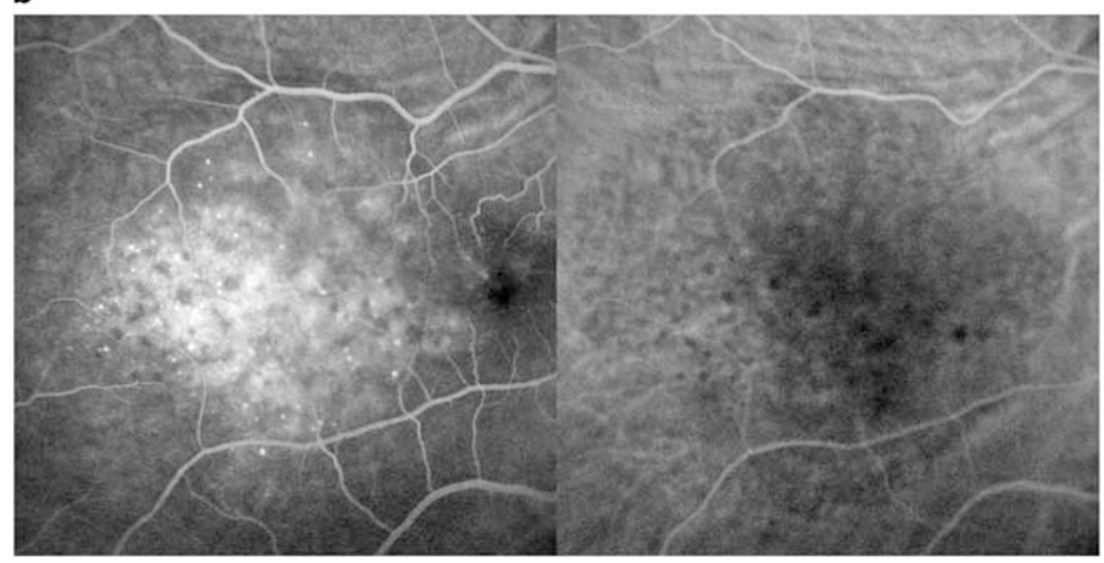

Figure 6 (a) Fundus autofluorescence image of the choroidal melanoma depicted in Figure 3a demonstrating a patchy pattern. (b) Mid-late phase FA/ICGA demonstrating hyperfluorescence and diffuse leakage (FA) (left) and hypofluorescence (ICGA) (right) in the tumour. 

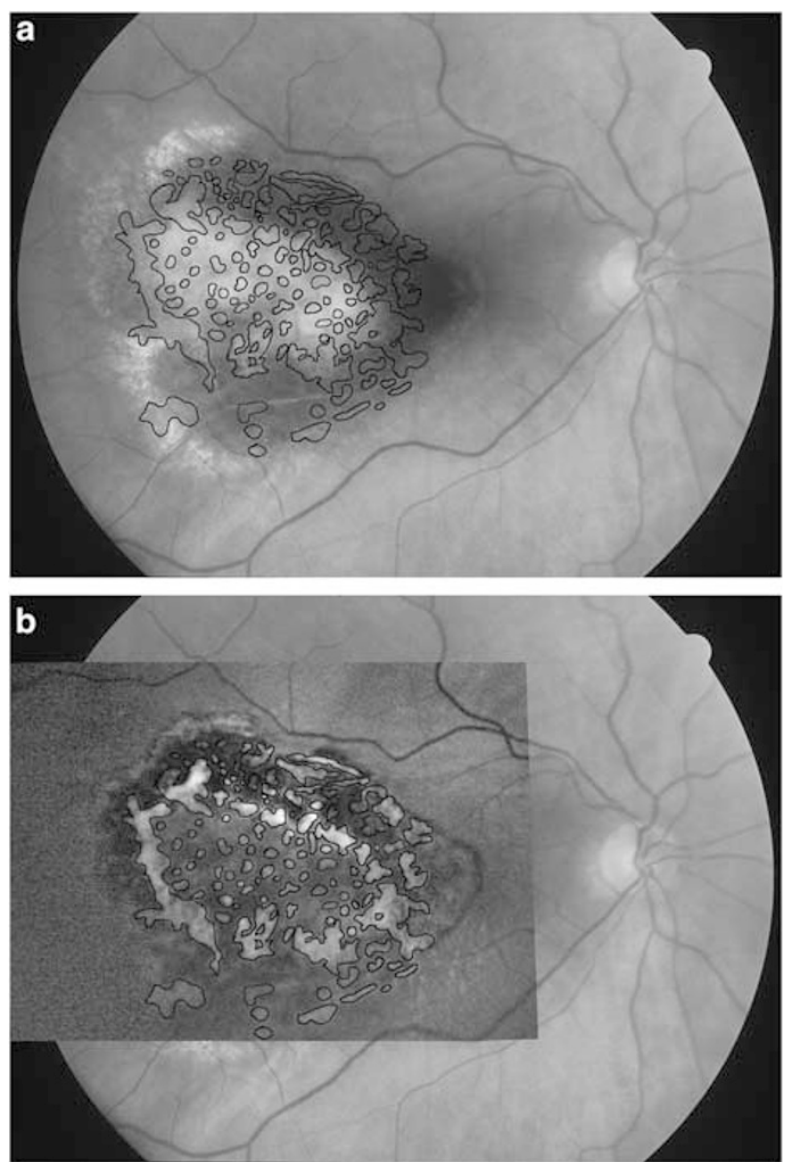

Figure 7 (a) Colour fundus photograph of the pigmented choroidal melanoma depicted in Figure 3a three months after treatment. Extensive lipofuscin accumulation (black outlines) overlying the lesion can be seen. (b) The composite FAF-colour fundus picture of the same eye showing complete correlation between lipofuscin and increased autofluorescence pattern (black outlines).

tumour thickness $(P=0.00001)$ and increased tumour base diameter $(P=0.001){ }^{26}$

FAF imaging can also be used to follow-up the response to treatment in choroidal melanomas. We evaluated the post-treatment FAF imaging features of six patients with choroidal melanoma. ${ }^{29}$ Two out of six patients were treated with plaque radiotherapy alone and four patients with plaque radiotherapy in combination with transpupillary thermotherapy. At a median follow-up of 4.9 months (range 2-9) after treatment, all treated tumours had an increase in lipofuscin and hyperpigmentation over the lesion surface. ${ }^{29}$ In all six tumours, a complete correlation was found between increased FAF and lipofuscin after treatment (Figure 7a and b), whereas a partial correlation was observed between hyperpigmentation and increased FAF.
The increase of lipofuscin observed in melanomas after treatment might be secondary to the damage suffered by the RPE cells in the necrosing tumour. The increase in hyperpigmentation may be secondary to the increased metabolic activity of the RPE during the proliferation that follows destruction, similar to that seen after laser treatment. ${ }^{17}$

There are certain limitations to our observations. AF and angiography images were obtained using HRA cSLO in these studies. The HRA employs a confocal light detection unit, which has a pinhole aperture to suppress the light originating from retinal locations outside the focal plane. Therefore, when imaging studies are performed over elevated lesions, the out-of-focus regions either will not be visible or the image quality will be poor. ${ }^{30}$ It is important to remember this when looking at elevated fundus lesions. The lens is another potential source of AF in the eye. The confocal optics of the HRA effectively eliminates the FAF from the lens. Motion artifacts may lead to background noise resulting in aberrant FAF signals. Therefore, camera adjustment is crucial. Furthermore, a normative FAF database is not available. Consequently, the images from different cSLO equipment with different outputs cannot be reliably compared with each other. ${ }^{31}$ Other factors that may interfere with the FAF signals include the presence of subretinal fluid, intraretinal oedema, and presence of a pigment epithelial detachment over the lesion. ${ }^{32,33}$ These factors may result in partial or poor correlation between lipofuscin/melanolipofuscin and the increased FAF signal.

In conclusion, FAF associated with pigmented choroidal lesions can be attributed to mainly lipofuscin (orange pigment) but also to hyperpigmentation, drusen, and fibrous metaplasia. Whether the diffuse FAF pattern seen in choroidal melanomas portends a poor prognosis for metastasis remains unknown. A larger number of patients with longer follow-up are required to validate these observations.

\section{References}

1 Rovati L, Docchio F. Autofluorescence methods in ophthalmology. J Biomed Optics 2004; 9: 9-21.

2 Eldred GE, Katz ML. Fluorophores of the retinal pigment epithelium. Exp Eye Res 1988; 47: 71-86.

3 Delori FC, Dorey CK, Staurenghi G, Arend O, Goger DG, Weiter JJ. In vivo fluorescence of the ocular fundus exhibits retinal pigment epithelium lipofuscin characteristics. Invest Ophthalmol Vis Sci 1995; 36: 718-729.

4 Holz FG, Schuett F, Kopitz J, Eldred GE, Kruse FE, Völcker HE et al. Inhibition of lysosomal degradative functions in RPE cells by a retinoid component of lipofuscin. Invest Ophthalmol Vis Sci 1999; 40: 737-743. 
5 Von Rückmann A, Fitzke FW, Bird AC. Fundus autofluorescence in age-related macular disease imaged with a scanning laser ophthalmoscope. Invest Ophthalmol Vis Sci 1997; 38: 478-486.

6 Lois N, Owens SL, Coco R, Hopkins J, Fitzke FW, Bird AC. Fundus autofluorescence in patients with age-related macular degeneration and high risk of visual loss. Am J Ophthalmol 2002; 133: 341-349.

7 Einbock W, Moessner A, Schnurrbusch UE, Holz FG, Wolf S, FAM Study Group. Changes in fundus autofluorescence in patients with age-related maculopathy. Correlation to visual function: a prospective study. Graefes Arch Clin Exp Ophthalmol 2005; 243: 300-305.

8 Solbach U, Keilhauer C, Knabben H, Wolf S. Imaging of retinal autofluorescence in patients with age-related macular degeneration. Retina 1997; 17: 385-389.

9 Bindewald A, Bird A, Dandekar SS, Dolar-Szczasny J, Dreyhaupt J, Fitzke FW et al. Classification of fundus autofluorescence patterns in early age-related macular disease. Invest Ophthalmol Vis Sci 2005; 46: 3309-3314.

10 Framme C, Bunse A, Sofroni R, Thalhammer T, Walter A, Sachs HG et al. Fundus autofluorescence before and after photodynamic therapy for choroidal neovascularization secondary to age-related macular degeneration. Ophthalmic Surg Lasers Imaging 2006; 37: 406-414.

11 Dandekar SS, Jenkins SA, Peto T, Scholl HP, Sehmi KS, Fitzke FW et al. Autofluorescence imaging of choroidal neovascularization due to age-related macular degeneration. Arch Ophthalmol 2005; 123: 1507-1513.

12 Von Rückman A, Fitzke FW, Fan J, Halfyard A, Bird AC. Abnormalities of fundus autofluorescence in central serous retinopathy. Am J Ophthalmol 2002; 133: 780-786.

13 Von Rückmann A, Fitzke FW, Bird AC. In vivo fundus autofluorescence in macular dystrophies. Arch Ophthalmol 1997; 115: 609-615.

14 Lois N, Halfyard AS, Bird AC, Holder GE, Fitzke FW. Fundus autofluorescence in Stargardt macular dystrophyfundus flavimaculatus. Am J Ophthalmol 2004; 138: 55-63.

15 Sawa M, Ober MD, Freund KB, Spaide RF. Fundus autofluorescence in patients with pseudoxanthoma elasticum. Ophthalmology 2006; 113: 814-820.

16 Sunness JS, Ziegler MD, Applegate CA. Issues in quantifying atrophic macular disease using retinal autofluorescence. Retina 2006; 26: 666-672.

17 Framme C, Brinkmann R, Birngruber R, Roider J. Autofluorescence imaging after selective RPE laser treatment in macular diseases and clinical outcome: a pilot study. Br J Ophthalmol 2002; 86: 1099-1106.

18 Font RL, Zimmerman LE, Armaly MF. The nature of the orange pigment over a choroidal melanoma. Arch Ophthalmol 1974; 91: 359-362.

19 Lindley TS, Irvine AR. Diagnostic significance of orange pigment accumulation over choroidal tumors. Am J Ophthalmol 1973; 76: 212-216.
20 Gündüz K, Pulido JS, Bakri SJ, Petit-Font E. Fundus autofluorescence in choroidal melanocytic lesions: A case series. Retina 2007; 27: 681-687.

21 The Laser Institute of America. American National Standards of the Safe Use of Laser. The American National Standards Institute: Toledo, OH, 1993: ANSI Z 136.1.1993.

22 Lavinsky D, Belfort RN, Navajas E, Torres V, Martins MC, Belfort Jr R. Fundus autofluorescence of choroidal nevus and melanoma. Br J Ophthalmol 2007; 91: 1299-1302. Epub 2007 Apr 12.

23 Sandby-Moller J, Thieden E, Philipsen PA, Heydenreich J, Wulf HC. Skin autofluorescence as a biological UVR dosimeter. Photodermatol Photoimmunol Photomed 2004; 20: 33-40.

24 Shields CL, Cater J, Shields JA, Singh AD, Santos MC, Carvalho C. Combination of clinical factors predictive of growth of small choroidal melanocytic tumors. Arch Ophthalmol 2000; 118: 360-364.

25 Shields CL, Shields JA, Kiratli H, De Potter P, Cater JR. Risk factors for growth and metastasis of small choroidal melanocytic lesions. Ophthalmology 1995; 102: 1351-1361.

26 Gündüz K, Pulido JS, Pulido JE, Link T. Correlation of fundus autofluorescence with fluorescein and indocyanine green angiography in choroidal melanocytic lesions. Retina 2008 July 3 [e-pub ahead of print].

27 Bindewald A, Bird A, Dandekar SS, Dolar-Szczasny J Dreyhaupt J, Fitzke FW et al. Classification of fundus autofluorescence patterns in early age-related macular disease. Invest Ophthalmol Vis Sci 2005; 46: 3309-3314.

28 Mueller AJ, Dirk-Uwe B, Folberg R, Mehaffey MG, Boldt $\mathrm{HC}$, Meyer $\mathrm{M}$ et al. Imaging the microvasculature of choroidal melanomas with confocal indocyanine green scanning laser ophthalmoscopy. Arch Ophthalmol 1998; 116: 31-39.

29 Gündüz K, Pulido JS, Bakri SJ, Amselem L, Petit-Fond E, Link T. Fundus autofluorescence in choroidal melanocytic lesions and the effect of treatment. Trans Am Ophthal Soc 2007; 105: 172-179.

30 Bartsch DG, Freeman WR. Scanning laser ophthalmoscopy. In: Ciulla TA, Regillo CD, Harris A (eds). Retina and Optic Nerve Imaging. Lippincott Williams and Wilkins: Philadelphia, 2003, pp 59-76.

31 Hopkins J, Walsh A, Chakravarthy U. Fundus autofluorescence in age-related macular degeneration: an epiphenomenona? Invest Ophthalmol Vis Sci 2006; 47: 22692271.

32 Karadimas P, Bouzas EA. Fundus autofluorescence imaging in serous and drusenoid pigment epithelial detachments associated with age-related macular degeneration. Am J Ophthalmol 2005; 140: 1163-1165.

33 Delori FA, Fleckner MR, Goger DG, Weiter JJ, Dorey CK. Autofluorescence distribution associated with drusen in age-related macular degeneration. Invest Ophthalmol Vis Sci 2000; 41: 496-504. 